\title{
ON MAXIMA AND LADDER PROCESSES FOR A DENSE CLASS OF LÉVY PROCESS
}

\author{
MARTIJN PISTORIUS, ${ }^{*}$ King's College London
}

\begin{abstract}
In this paper, we present an iterative procedure to calculate explicitly the Laplace transform of the distribution of the maximum for a Lévy process with positive jumps of phase type. We derive error estimates showing that this iteration converges geometrically fast. Subsequently, we determine the Laplace transform of the law of the upcrossing ladder process and give an explicit pathwise construction of this process.

Keywords: Lévy process; first passage; ladder process; Wiener-Hopf factorization; phasetype distribution; Markov additive process; nonlinear iteration
\end{abstract}

2000 Mathematics Subject Classification: Primary 60G51; 60K15; 60J25

\section{Introduction}

A Lévy process is a stochastic process with homogeneous, independent increments and right-continuous paths with left limits. In mathematical finance several authors, among them Barndorff-Nielssen [7], Eberlein and Keller [11] and Carr et al. [10], have proposed particular Lévy processes as models for the evolution of log-asset prices. In this setting, the need to price exotic financial instruments that depend on the pathwise maximum or minimum, such as barrier options or equity default swaps (see, e.g. [6] and [13]), leads to the search for an analytically tractable characterization of the distribution of the running maximum of the underlying Lévy process. One approach to such a characterization starts from the famed Wiener-Hopf factorization that relates the double Laplace transforms of the distributions of the running maximum and the running infimum of a Lévy process with its characteristic exponent. In general, this factorization is given by an expression in terms of double integrals involving the one-dimensional distributions of the Lévy process (see, e.g. [8, Theorem VI.5, p. 160]). Only for Lévy processes with a special form of the Lévy measure is the Wiener-Hopf factorization known analytically in terms of the characteristics of the Lévy process, for example if the Lévy measure has support on a half-axis (see, e.g. the comprehensive review by Bingham [9] or Bertoin [8, Chapter VII, pp. 187-192] for this case) or if the density of the Lévy measure on the positive half-axis is an exponential [13], a mixture of exponentials [15], or of phase type [5].

In view of the facts that, for a general Lévy process $X$, the Wiener-Hopf factorization is not available analytically and that $X$ can be approximated in law arbitrarily closely by a Lévy process whose jumps follow a phase-type distribution (see, e.g. [5, Proposition 1, p. 82]), a way to proceed is to approximate the first passage time distribution of $X$ by computing it for an approximating phase-type Lévy process $Z$. This strategy was pursued in [6] to price equity default swaps driven by the Carr-Geman-Madan-Yor Lévy process. In Section 2, we provide a mathematical proof showing that the approximation of $X$ indeed carries over to that of the distribution of the first passage time of $X$ over some level.

Received 21 January 2005; revision received 9 September 2005.

* Postal address: Department of Mathematics, King's College London, Strand, London WC2R 2LS, UK.

Email address: pistoriu@mth.kcl.ac.uk 
In this paper, we shall focus on a Lévy process $Z$ whose positive jumps follow a phasetype distribution. In [16] it was shown that the distribution of the running maximum of $Z$ at an exponential time follows a certain phase-type distribution. We derive an explicit characterization of the parameters of this phase-type distribution by showing that they are given in terms of the solution to a certain fixed-point equation $\boldsymbol{\eta}=\psi_{a}(\boldsymbol{\eta})$. We subsequently show that this fixed-point equation has a unique subprobability vector as a solution, which can be found by iteration: the sequence $\boldsymbol{\eta}^{(n)}=\psi_{a}\left(\boldsymbol{\eta}^{(n-1)}\right)$, resulting from iterating $\psi_{a}$, is shown to converge geometrically fast to the solution to the fixed-point equation. In the matrix-geometric setting, fixed-point equations are often solved by employing monotonicity properties (see [17] and [18]). Asmussen [2] constructed a probabilistic coupling argument to analyse a fixed-point equation similar to ours in a random walk setting. Our error estimates are mainly based on a contraction argument.

Classically, the upcrossing ladder process is used to describe the dynamics of the running maximum of a Lévy process. The ladder process is closely related to the times at which a new maximum is attained and the value of this maximum (for a precise description, see [8, Chapter VI, pp. 155-159]). A second contribution of this paper is a characterization of the upcrossing ladder process of $Z$. We derive an explicit expression for the joint moment generating function of the ladder height and ladder time processes and subsequently describe an explicit construction of these processes in terms of the aforementioned vector $\boldsymbol{\eta}$ and the characteristics of $Z$. This description could in principle be used to simulate paths of the running maximum; a numerical investigation of this idea is left for future research. The proofs of the described results rely on the extension of the classical Wiener-Hopf factorization results of London et al. [14] to a class of spectrally negative Markov additive processes, and on an embedding of the Lévy process $Z$ into a spectrally negative Markov additive process. The technique of embedding was first proposed by Asmussen [3] in the setting of regime-switching Brownian motion.

The rest of the paper is organized as follows. In Section 2, the notation is set and preliminaries are given. In Section 3, the main results of the paper are stated. Sections 4 and 5 are devoted to the proof of the Wiener-Hopf factorization and the study of the fixed-point equation $\boldsymbol{\eta}=\psi_{a}(\boldsymbol{\eta})$, respectively.

\section{Preliminaries}

We start by recalling the definition and some properties of a phase-type distribution, and refer the reader to [17] and [1] for background on phase-type distributions.

A distribution $F$ on $(0, \infty)$ is called phase type if it is the distribution of the absorption time $\zeta$ in a finite-state continuous-time Markov process $J=\left\{J_{t}\right\}_{t \geq 0}$ with one state, $\Delta$, absorbing and the remaining ones, denoted $1, \ldots, m$, transient. That is, $F(t)=\mathrm{P}(\zeta \leq t)$, where $\zeta=\inf \left\{s>0: J_{s}=\Delta\right\}$. The parameters are $m$, the restriction, $\boldsymbol{T}$, of the full intensity matrix to the $m$ transient states, and the initial probability (row) vector $\boldsymbol{\alpha}=\left(\alpha_{1}, \ldots, \alpha_{m}\right)$, where $\alpha_{i}=\mathrm{P}\left(J_{0}=i\right)$. For any $i=1, \ldots, m$, let $t_{i}$ be the intensity of a transition $i \rightarrow \Delta$ and write $\boldsymbol{t}=\left(t_{1}, \ldots, t_{m}\right)^{\top}$ for the (column) vector of such intensities. Note that $\boldsymbol{t}=-\boldsymbol{T} \mathbf{1}$, where $\mathbf{1}$ denotes a column vector of $1 \mathrm{~s}$. It follows that the cumulative distribution $F$ is given by

$$
1-F(x)=\alpha \mathrm{e}^{T x} \mathbf{1},
$$

the density is $f(x)=\boldsymbol{\alpha} \mathrm{e}^{\boldsymbol{T} x} \boldsymbol{t}$, and the Laplace transform is given by

$$
\hat{F}[s]=\int_{0}^{\infty} \mathrm{e}^{-s x} F(\mathrm{~d} x)=\boldsymbol{\alpha}(s \boldsymbol{I}-\boldsymbol{T})^{-1} \boldsymbol{t},
$$


where $\boldsymbol{I}$ is the $m \times m$ identity matrix. Note that $\hat{F}[s]$ can be extended to the complex plane except at a finite number of poles (the eigenvalues of $\boldsymbol{T}$ ). Throughout, we shall assume that representation (1) of the distribution function $F$ is minimal, that is, that there exists no number $k, k<m, k$-vector $\boldsymbol{b}$, and $k \times k$ matrix $\boldsymbol{G}$ such that $1-F(x)=\boldsymbol{b} \mathrm{e}^{\boldsymbol{G} x} \mathbf{1}$.

On some probability triplet $(\Omega, \mathcal{F}, \mathrm{P})$, let $X$ be a Lévy process of the form

$$
X_{t}=X_{t}^{(+)}+X_{t}^{(-)},
$$

where $X^{(-)}=\left\{X_{t}^{(-)}, t \geq 0\right\}$ is a Lévy process without positive jumps and $X^{(+)}=\left\{X_{t}^{(+)}\right.$, $t \geq 0\}$ is an independent compound Poisson process with intensity $\lambda^{(+)}$and jumps of phase type with parameter triple $\left(m^{(+)}, \boldsymbol{T}^{(+)}, \boldsymbol{\alpha}^{(+)}\right)$. In the sequel, we exclude the case in which $X^{(-)}$ has monotone paths. We define by $\kappa(s) \equiv \kappa_{X}(s)=\log \mathrm{E}\left[\mathrm{e}^{s X_{1}}\right]$ the Lévy exponent of $X$, which is well defined at least for those $s$ with $\operatorname{Re}(s)=0$ and which is in this case given by

$$
\kappa(s)=\kappa_{X^{(-)}}(s)+\lambda^{(+)}\left(\hat{F}^{(+)}[-s]-1\right),
$$

where $\kappa_{X^{(-)}}$denotes the Lévy exponent of $X^{(-)}$and $\hat{F}^{(+)}[s]=\boldsymbol{\alpha}^{(+)}\left(s \boldsymbol{I}-\boldsymbol{T}^{(+)}\right)^{-1} \boldsymbol{t}$ is the Laplace transform of $F^{(+)}$. Note that $\kappa$ can be analytically extended to the positive half-plane except at a finite number of poles (the eigenvalues of $-\boldsymbol{T}^{(+)}$) and that we also denote this extension by $\kappa$.

Write $T^{+}(k) \equiv T^{+}(k)(X)$ for the first passage time of $X$ over the level $k$, i.e.

$$
T^{+}(k)=\inf \left\{t \geq 0: X_{t}>k\right\},
$$

and denote by $O^{+}(k) \equiv O^{+}(k)(X)=X_{T^{+}(k)}-k$ the corresponding overshoot of $X$. Our first result shows that convergence in law of the Lévy processes $X^{n}$ carries over to the stopping times $T^{+}(k)\left(X^{n}\right)$ and the overshoots $O^{+}(k)\left(X^{n}\right)$.

Proposition 1. Let $\left(X^{n}\right)_{n}$ and $X$ be Lévy processes such that $X^{n} \rightarrow X$ in law. Then, as $n \rightarrow \infty$,

$$
\left(T^{+}(k)\left(X^{n}\right), O^{+}(k)\left(X^{n}\right)\right) \stackrel{\mathrm{D}}{\rightarrow}\left(T^{+}(k)(X), O^{+}(k)(X)\right),
$$

where $\stackrel{\mathrm{D}}{\rightarrow}$ ' denotes convergence in distribution.

Before giving the proof, we first review some facts about the Wiener-Hopf factorization of Lévy processes. For more background, we refer the reader to [9] or [8], and references therein. Denote by $\mathcal{I}^{(+)}=\left\{i: \operatorname{Re}\left(\rho_{i}(a)\right)>0\right\}$ the set of indices corresponding to the roots, $\rho_{i}(a)$, of the Cramèr-Lundberg equation

$$
\kappa(\rho)=\kappa_{X}(\rho)=a
$$

with positive real parts. Let $x \wedge 0=\min \{x, 0\}$ and $x \vee 0=\max \{x, 0\}$, and write $M_{t}=$ $\sup _{s \leq t}\left(X_{t} \vee 0\right)$ and $I_{t}=\inf _{s \leq t}\left(X_{t} \wedge 0\right)$ for the running supremum and infimum of $X$ up to time $t$, respectively. Similarly, write $M_{t}^{(-)}$and $I_{t}^{(-)}$for the corresponding quantities of $X^{(-)}$. Denote by $e(a)$ an independent exponential random variable with mean $a^{-1}$. For $s$ with $\operatorname{Re}(s) \geq 0$, set

$$
\varphi_{a}^{-}(s)=\mathrm{E}\left[\exp \left(s I_{e(a)}\right)\right], \quad \varphi_{a}^{+}(-s)=\mathrm{E}\left[\exp \left(-s M_{e(a)}\right)\right] .
$$

If $X$ is a Lévy process of the form (2), the phase-type representation is minimal, and $a>0$, then, for $\operatorname{Re}(s) \leq 0, \varphi_{a}^{+}$is explicitly given by

$$
\varphi_{a}^{+}(s)=\frac{\operatorname{det}\left(-s \boldsymbol{I}-\boldsymbol{T}^{(+)}\right)}{\operatorname{det}\left(-\boldsymbol{T}^{(+)}\right)} \frac{\prod_{i \in \mathcal{X}^{(+)}}\left(-\rho_{i}(a)\right)}{\prod_{i \in \mathcal{X}^{(+)}}\left(s-\rho_{i}(a)\right)}
$$


(as shown in [5]), where the first factor is to be taken to equal 1 if $X$ has no positive jumps. The following Wiener-Hopf identity (see, e.g. [9, Theorem 1e, p. 708]) links the joint distribution of the first time of crossing the level $k$ and the corresponding overshoot $\left(T^{+}(k), O^{+}(k)\right)$ to the Wiener-Hopf factor $\varphi_{a}^{+}$:

$$
\mathrm{E}\left[\exp \left(-a T^{+}(e(\lambda))-\mu O_{e(\lambda)}^{+}\right)\right]=\frac{\lambda}{\lambda-\mu}\left[1-\frac{\varphi_{a}^{+}(-\lambda)}{\varphi_{a}^{+}(-\mu)}\right] .
$$

We have now made all preparations necessary for the proof of Proposition 1.

Proof of Proposition 1. Let $D[0, \infty)$ denote the space of càdlàg functions (i.e. those that are right continuous with left limits) on $[0, \infty)$ equipped with the Skorokhod topology. From Proposition VI.2.4 of [12, p. 303] it follows that the map $M_{t}: D[0, \infty) \rightarrow \mathbb{R}$ given by $f \mapsto M_{t}(f):=\sup _{0 \leq s \leq t}(f(s) \vee 0)$ is continuous for every $t$ for which $\Delta f(t):=f(t)-$ $f\left(t^{-}\right)=0$. Since with probability 1 a Lévy process is continuous at a fixed time $t$ (see, e.g. [8, Proposition I.7, p. 21]), it follows that $M_{t}\left(X^{n}\right) \rightarrow M_{t}(X)$ in law for a fixed $t \geq 0$ (cf. [12, Result VI.3.8, p. 312]). This implies that the moment generating function of $M_{e(q)}\left(X^{n}\right)$ converges to the moment generating function of $M_{e(q)}(X)$. By the Wiener-Hopf identity (3) and the extended continuity theorem, it then follows that the joint Laplace transform of $\left(T^{+}(k)\left(X^{n}\right), O^{+}(k)\left(X^{n}\right)\right)$ converges to that of $\left(T^{+}(k)(X), O^{+}(k)(X)\right)$.

Closely related to the supremum process $M$ are the ascending ladder time and the ladder height processes, which are Lévy processes. The ladder time is the right-inverse, $L_{t}^{-1}=$ $\inf \left\{s \geq 0: L_{s}>t\right\}$, of the local time $L$, which we shall choose to be equal to $L=M^{\mathrm{c}}$, the continuous part of the supremum process $M$. The ladder height process is given by $H=M_{L^{-1}}$, the supremum at the ladder time. We write $\kappa^{+}$for the joint characteristic exponent of the ladder process $\left(L^{-1}, H\right)$ :

$$
\exp \left(-\kappa^{+}(a, s)\right)=\mathrm{E}\left[\exp \left(-a L^{-1}(1)-s H(1)\right)\right], \quad a, s>0 .
$$

\section{Maxima and ladder processes}

Before stating our main results, we introduce some further notation. Let $\&$ be the set of subprobability vectors in $\mathbb{R}^{m+1}$ with $m=m^{(+)}$, and consider the fixed-point equation $\eta=$ $\psi_{a}(\boldsymbol{\eta})$, where $\psi_{a}: s \rightarrow \mathbb{R}^{m+1}$ is defined by

$$
\psi_{a}: \boldsymbol{\eta} \mapsto \frac{\lambda^{(+)}}{a+\lambda^{(+)}}\left(0, \boldsymbol{\alpha}^{(+)}\right) \varphi_{a, X^{(-)}}^{(-)}\left(-\boldsymbol{M}^{(+)}-\boldsymbol{m}^{(+)} \boldsymbol{\eta}\right)
$$

Here $\varphi_{a, X^{(-)}}^{(-)}(-\boldsymbol{Q})=\int_{0}^{\infty} \mathrm{e}^{\boldsymbol{Q} x} \mathrm{P}\left(-I_{e(a)}^{(-)} \in \mathrm{d} x\right)$ and

$$
\boldsymbol{M}^{(+)}=\left(\begin{array}{cc}
-\Phi\left(a+\lambda^{(+)}\right) & \mathbf{0} \\
\boldsymbol{t}^{(+)} & \boldsymbol{T}^{(+)}
\end{array}\right), \quad \boldsymbol{m}^{(+)}=-\boldsymbol{M}^{(+)} \mathbf{1}=\left(\begin{array}{c}
\Phi\left(a+\lambda^{(+)}\right) \\
\mathbf{0}
\end{array}\right),
$$

where $\Phi(q)$ denotes the largest root of $\kappa_{X^{(-)}}(s)=q$.

Lemma 1. Let $a \geq 0$. The function $\psi_{a}$ maps $\&$ into $\&$ and the fixed-point equation $\boldsymbol{\eta}=\psi_{a}(\boldsymbol{\eta})$ has a unique solution $\eta_{a}$.

Let $\mathbf{1}_{0}^{\top}$ denote the row vector with a 1 in the first position and 0 s elsewhere, and write $f_{a}$ for the function that maps $\eta \in \&$ to

$$
f_{a}(\boldsymbol{\eta})=a^{-1} \mathbf{1}_{0}^{\top} \exp \left(\left(\boldsymbol{M}^{(+)}+\boldsymbol{m}^{(+)} \boldsymbol{\eta}\right) k\right) \mathbf{1}, \quad k \geq 0 .
$$


The Laplace transform of $\mathrm{P}\left(M_{t}>k\right)=\mathrm{P}\left(T^{+}(k)<t\right)$ can be expressed in terms of the vector $\eta_{a}$ as follows [16]:

$$
\int_{0}^{\infty} \mathrm{e}^{-a t} \mathrm{P}\left(M_{t}>k\right) \mathrm{d} t=f_{a}\left(\boldsymbol{\eta}_{a}\right)
$$

The next result shows how $f_{a}\left(\boldsymbol{\eta}_{a}\right)$ can be computed explicitly.

Theorem 1. Let $\boldsymbol{\eta}^{(n+1)}=\psi_{a}\left(\boldsymbol{\eta}^{(n)}\right)$ for $n \geq 0$.

(i) If $\boldsymbol{\eta}^{(0)}=\mathbf{0}$ then $f_{a}\left(\boldsymbol{\eta}^{(n)}\right) \uparrow f_{a}\left(\boldsymbol{\eta}_{a}\right)$ as $n \rightarrow \infty$.

(ii) If $\hat{F}^{(+)}[-\Phi(0)]<1+a / \lambda^{(+)}, a>0$, and $\boldsymbol{\eta}^{(0)} \in \delta$, then

$$
\left\|f_{a}\left(\boldsymbol{\eta}_{a}\right)-f_{a}\left(\boldsymbol{\eta}^{(n)}\right)\right\| \leq C \varepsilon^{n-1}\left\|\boldsymbol{\eta}_{a}-\boldsymbol{\eta}^{(0)}\right\|,
$$

where $\|\boldsymbol{\eta}\|=\sum_{i}\left\{\left|\boldsymbol{\eta}_{i}\right|\right\}, C=k \Phi(a) / a$, and $\varepsilon<1$ is explicitly given by

$$
\varepsilon=\lambda^{(+)} \hat{F}^{(+)}[-\Phi(0)] \frac{a-(\Phi(a)-\Phi(0)) \kappa_{X^{(-)}}^{\prime}\left(\Phi(0)^{+}\right)}{a\left(\lambda^{(+)}+a\right)} .
$$

The next result gives an explicit formula for the cumulant of the upcrossing ladder process and describes a pathwise construction of the ladder process.

Theorem 2. (i) The cumulant, $\kappa^{+}$, of $\left(L^{-1}, H\right)$ is given by

$$
\kappa^{+}(a, s)=\frac{\prod_{i \in \mathcal{I}^{(+)}}\left(s+\rho_{i}(a)\right)}{\operatorname{det}\left(s \boldsymbol{I}-\boldsymbol{T}^{(+)}\right)}
$$

for $s$ with $\operatorname{Re}(s)=0$ and $a \geq 0$, where the denominator is taken to equal 1 if $\lambda^{(+)}=0$.

(ii) The components of the bivariate subordinator $\left(L^{-1}, H\right)$ are equal to

$$
L_{t}^{-1}=Z_{t}+J_{t}^{1}, \quad H_{t}=t+J_{t}^{2},
$$

where $Z$ is a subordinator with cumulant $\Phi$ and $J=\left(J^{1}, J^{2}\right)$ is a bivariate compound Poisson process, independent of $Z$, with arrival rate $\Phi\left(\lambda^{(+)}\right)$and jump distribution $G$ with

$$
\iint_{[0, \infty)^{2}} \mathrm{e}^{-a x-s y} G(\mathrm{~d} x, \mathrm{~d} y)=\Phi\left(\lambda^{(+)}\right)^{-1}\left[\kappa^{+}(a, s)-s-\Phi(a)+\Phi\left(\lambda^{(+)}\right)\right] .
$$

In particular, the marginal law of the jumps, $\tilde{U}_{n}$, of $J^{2}$ is given by

$$
\mathrm{P}\left(\tilde{U}_{n} \in \mathrm{d} y\right)=\eta_{0}\left(\delta_{0}(\mathrm{~d} y), \exp \left(\boldsymbol{T}^{(+)} y\right) \boldsymbol{t}^{(+)} \mathrm{d} y\right),
$$

where $\delta_{0}$ is the delta measure at 0 .

Example 1. If there are no positive jumps $\left(\lambda^{(+)}=0\right)$ and $-X^{(-)}$is not a subordinator, we find that (see, e.g. [8])

$$
\kappa^{+}(a, s)=s+\Phi(a),
$$

where $\Phi(a)$ is the unique positive real root of $\kappa_{X^{(-)}}(s)=a$.

\section{Matrix Wiener-Hopf factorizations}

This section is devoted to the proof of Theorem 2. In preparation, we shall first extend the matrix Wiener-Hopf factorization results of London et al. [14] to a class of spectrally negative Markov additive processes. As a by-product we shall also obtain a short proof of (6). 


\subsection{Matrix factorization of Markov additive processes}

Let $Y$ be an irreducible Markov process with finite state space $E \cup\{\Delta\}$, where $E=$ $\{0,1, \ldots, m\}$ and $\Delta$ is an absorbing graveyard state. Denote the generator of $Y$ restricted to $E$ by $\boldsymbol{Q}=\left(q_{i j}\right)_{i, j=0}^{m}$. Letting $X^{(-)}$be the spectrally negative Lévy process of (2) and denoting by $v$ and $\sigma$ functions that map $\Delta$ to $\partial$ (an isolated point acting as a graveyard state) and, respectively, $E$ to $\mathbb{R}$ and $[0, \infty)$, we now consider the additive functional $A=\left\{A_{t}, t \geq 0\right\}$ with

$$
A_{t}=A_{0}+\int_{0}^{t} \sigma\left(Y_{s}\right) \mathrm{d} X_{s}^{(-)}+\int_{0}^{t} v\left(Y_{s}\right) \mathrm{d} s .
$$

Note that this model reduces to a spectrally one-sided Lévy process if $v \equiv 0, \sigma$ is constant, and $Y$ is recurrent. Next, we define $\tilde{Y}^{+}$to be the upcrossing ladder process of $Y$, that is,

$$
\tilde{Y}_{t}^{+}=Y\left(\gamma_{t}\right), \quad \text { where } \gamma_{t}=\inf \left\{s \geq 0: A_{s}>t\right\} .
$$

It is easily verified that the time-changed process $\tilde{Y}^{+}$is again a Markov process; we denote its generator by $\boldsymbol{Q}^{+}$. This subsection is devoted to a characterization of its form. Let $\boldsymbol{K}(\sigma,-\boldsymbol{G})$ be the matrix whose $i$ th row is given by $\mathbf{1}_{i}^{\top} \kappa_{X^{(-)}}(-\sigma(i) \boldsymbol{G})$. Here $\mathbf{1}_{i}^{\top}$ is the row vector whose $i$ th component is 1 and whose other components are 0 , and $\kappa_{X^{(-)}}$is given by

$$
\kappa_{X^{(-)}}(-\boldsymbol{G})=\frac{s^{2}}{2} \boldsymbol{G}^{2}-c \boldsymbol{G}+\int_{-\infty}^{0}\left(\mathrm{e}^{-\boldsymbol{G} x}-\boldsymbol{I}+x \boldsymbol{G} 1_{\{|x|<1\}}\right) \nu(\mathrm{d} x)
$$

(using the Lévy-Khinchin formula), where $c$ is some constant, $v$ is the Lévy measure, $s$ is the Gaussian coefficient of $X^{(-)}$, and $1_{\{|x|<1\}}$ is the indicator of the set $\{|x|<1\}$.

We restrict ourselves to the case in which the function $v$ is positive with $v(i)>0$ for each $i \in E$ for which $\sigma(i)=0$, to ensure that $A$ can increase in each state in $E$ (recalling that we exclude the case in which $X^{(-)}$has monotone paths). Denote by $\mathcal{Q}(n)$ the set of irreducible generator matrices and let $\boldsymbol{V}$ be the $|E| \times|E|$ diagonal matrix with elements $v(i)$.

Proposition 2. (i) The generator matrix, $\mathbf{Q}^{+}$, of the process $\tilde{Y}^{+}$, defined in (9), solves the equation

$$
\boldsymbol{K}(\sigma,-\boldsymbol{G})+\boldsymbol{Q}=\boldsymbol{V} \boldsymbol{G}
$$

(ii) If $\boldsymbol{Q}$ is transient or if $\boldsymbol{Q}$ is recurrent and $\sup _{t} A_{t}=\infty$ almost surely, then $\boldsymbol{Q}^{+}$is the unique $\boldsymbol{G} \in \mathcal{Q}(|E|)$ that solves (11).

Proof. (i) Define the function $f: E \times \mathbb{R} \rightarrow \mathbb{R}$ by $f(j, x)=\mathrm{E}_{j, x}\left[h\left(\tilde{Y}_{k}^{+}\right) 1_{\left\{\gamma_{k}<\infty\right\}}\right]$, where $h$ is any function on $E$ and $\mathrm{E}_{j, x}$ denotes the expectation under the measure $\mathrm{P}$, conditioned on $\left\{Y_{0}=j, A_{0}=x\right\}$. Since $\tilde{Y}^{+}$is a Markov process with generator $\boldsymbol{Q}^{+}$, the function $f$ can be expressed as

$$
f(i, x)=\mathbf{1}_{i}^{\top} \exp \left(\boldsymbol{Q}^{+}(k-x)\right) \boldsymbol{h}, \quad i \in E, x \leq k .
$$

However, it is straightforward to check that $f\left(Y_{t \wedge \gamma_{k}}, A_{t \wedge \gamma_{k}}\right)$ is a martingale and, from Itô's lemma, we find that $\boldsymbol{f}=(f(i, u), i \in E)$ satisfies

$$
\Gamma(\sigma(i) f(i, u))+v(i) f^{\prime}(i, u)+\sum_{j \in E} q_{i j}(f(j, u)-f(i, u))=0
$$

for $i \in E$ and $u<k$, where $\Gamma$ denotes the infinitesimal generator of the process $X^{(-)}$:

$$
\Gamma f(x)=\frac{s^{2}}{2} f^{\prime \prime}(x)+c f^{\prime}(x)+\int_{-\infty}^{0}\left(f(x+y)-f(x)-y f^{\prime}(x) 1_{\{|y|<1\}}\right) v(\mathrm{~d} y) .
$$


Here a prime denotes differentiation. By substituting (12) into (13) and using the fact that $\boldsymbol{h}$ is arbitrary, we conclude that $\boldsymbol{Q}^{+}$solves (11).

(ii) Now we turn to the proof of the uniqueness of the solution to (11). To this end, let $\boldsymbol{G} \in \mathcal{Q}(|E|)$ be another solution to (11) and define the function $\tilde{f}$ by replacing $\boldsymbol{Q}_{+}$by $\boldsymbol{G}$ in (12); by an application of Itô's lemma it then follows that $\tilde{f}\left(Y_{t}, A_{t}\right)$ is a local martingale that is bounded on $\left[0, \gamma_{k}\right]$, and invoking the optional stopping theorem yields

$$
\begin{aligned}
\tilde{f}(j, x) & =\mathrm{E}_{j, x}\left[\tilde{f}\left(Y_{t \wedge \gamma_{k}}, A_{t \wedge \gamma_{k}}\right)\right] \\
& =\mathrm{E}_{j, x}\left[\tilde{f}\left(\tilde{Y}_{k}^{+}, A_{\gamma_{k}}\right) 1_{\left\{\gamma_{k}<\infty\right\}}\right]+\lim _{t \rightarrow \infty} \mathrm{E}_{j, x}\left[\tilde{f}\left(Y_{t}, A_{t}\right) 1_{\left\{\gamma_{k}=\infty\right\}}\right] .
\end{aligned}
$$

By definition of $\tilde{f}$ and the absence of positive jumps of $A$, the first expectation in (14) is equal to $f(j, x)$. Note that second term in (14) vanishes if $\boldsymbol{Q}$ is transient or $\boldsymbol{Q}$ is recurrent and $\sup _{t} A_{t}=\infty$. Indeed, in the latter case $\gamma_{k}$ is almost surely finite, whereas in the former case $\mathrm{P}\left(Y_{t} \in E\right)$ converges to 0 . Thus, $f=\tilde{f}$ and statement (ii) follows.

\subsection{Matrix factorization of $X$}

To study properties of the Lévy process $X$, we follow some ideas of Asmussen [4] and embed $X$ into a Markov process $(A, Y)$. Informally, we obtain $A$ from $X$ by 'levelling out' the positive jumps into piecewise-linear parts of gradient 1; the process $Y$ is set equal to 0 if $X$ moves like $X^{(-)}$, and equal to the underlying phase process of an upward jump otherwise. More precisely, consider the special case of the above additive functional, (8), in which $Y$ starts from state 0 with probability 1 , i.e. $\mathrm{P}\left(Y_{0}=0\right)=1$, and the restriction of the intensity matrix of $Y$ to $\{0,1, \ldots, m\}$ is given by $\boldsymbol{Q}_{0}$, where, in block notation for $a \geq 0$,

$$
\boldsymbol{Q}_{a}=\left(\begin{array}{cc}
-\lambda^{(+)}-a & \lambda^{(+)} \boldsymbol{\alpha}^{(+)} \\
\boldsymbol{t}^{(+)} & \boldsymbol{T}^{(+)}
\end{array}\right) .
$$

Moreover, for $i \in E$ set $v(i)=1-\sigma(i)$ and $\sigma(i)=\delta_{0 i}$, the Kronecker delta at 0 , and let $T_{0}(t)=\int_{0}^{t} 1_{\left\{Y_{s}=0\right\}} \mathrm{d} s$ denote the local time spent by $Y$ at 0 , that is, the amount of time before time $t$ that $Y$ has spent in state 0 . Then we recover the original process, $X$, by time-changing $A$ with the inverse local time

$$
T_{0}^{-1}(u)=\inf \left\{t \geq 0: T_{0}(t)>u\right\} .
$$

Indeed, the independence of the increments of $X$ implies that $X$ is equal in law to $A \circ T_{0}^{-1}$. Exponential killing of the original Lévy process $X$ at rate $a$ can be incorporated by replacing $\boldsymbol{Q}_{0}$ by $\boldsymbol{Q}_{a}$, since all states of $Y$ other than 0 originate from positive jumps of $X$, meaning that the local time, $T_{0}$, spent by $A$ at 0 corresponds to the time-scale of $X$. We can derive the following result for the form of the generator of $\tilde{Y}^{+}$.

Proposition 3. The generator matrix, $\boldsymbol{Q}_{a}^{+}$, of the Markov chain $\tilde{Y}^{+}$in (9) that corresponds to the embedding of $X$ killed at rate $a$ is given by

$$
\boldsymbol{Q}_{a}^{+}=\boldsymbol{M}^{(+)}+\boldsymbol{m}^{(+)} \boldsymbol{\eta}_{a}
$$

where $\boldsymbol{M}^{(+)}$is given in (5) and $\boldsymbol{\eta}_{a} \in \&$ solves $\boldsymbol{\eta}=\psi_{a}(\boldsymbol{\eta})$.

Note that the distribution, (6), of $M_{e(a)}$ now follows as a direct consequence of Proposition 3:

$$
\mathrm{P}\left(M_{e(a)}>k\right)=\mathrm{P}\left(\tilde{Y}^{+}(k) \notin \Delta\right)=\mathbf{1}_{0}^{\top} \exp \left(\boldsymbol{Q}_{a}^{+} k\right) \mathbf{1} .
$$


In the proof of Theorem 2 we shall also give a probabilistic interpretation of $\boldsymbol{\eta}_{a}$ as the distribution of $Y$ at the end of an excursion of $A$ from its supremum.

Proof of Proposition 3. The proof consists of identifying the form of the generator from the version of (11) corresponding to the process, $(A, Y)$, that is the imbedding of $X$. From the description given above, we are thus led to consider (11) with $\Sigma$ the diagonal matrix with elements $\delta_{0 i}, i \in E ; \boldsymbol{V}=\boldsymbol{I}-\boldsymbol{\Sigma}$; and $\boldsymbol{Q}$ given by $\boldsymbol{Q}_{a}$ from (15). The form of the matrices $\boldsymbol{\Sigma}$ and $\boldsymbol{V}$ in conjunction with (11) then implies that all rows of $\boldsymbol{Q}_{a}^{+}$except the first are given (in block notation) by $\left(\boldsymbol{t}^{(+)}, \boldsymbol{T}^{(+)}\right)$. By rewriting (11), for the first row we obtain

$$
\left[\frac{1}{a+\lambda^{(+)}}\left(\kappa_{X^{(-)}}\left(-\boldsymbol{Q}_{a}^{+}\right)-\left(a+\lambda^{(+)}\right) \boldsymbol{I}\right)\right]_{1}=-\frac{\lambda^{(+)}}{a+\lambda^{(+)}}(0, \boldsymbol{\alpha}),
$$

where $\boldsymbol{M}_{1}$ denotes the first row of a generic matrix $\boldsymbol{M}$. Since the supremum, $M_{e(q)}^{(-)}$, of $X^{(-)}$ at an independent exponential time $e(q)$ has an exponential distribution with mean $\Phi(q)^{-1}$, it follows that, for $q>0$ and $\operatorname{Re}(s) \geq 0$,

$$
q^{-1}\left(\kappa_{X^{(-)}}(s)-q\right) \varphi_{q, X^{(-)}}^{-}(s)=\Phi(q)^{-1}(\Phi(q)-s),
$$

where $\varphi_{q, X^{(-)}}^{-}$is the moment generating function of the running infimum, $I_{e(q)}^{(-)}$, of $X^{(-)}$at $e(q)$. By the Cayley-Hamilton theorem, this relation remains valid with $s$ replaced by a nonnegativedefinite matrix (and thus, in particular, with $s$ replaced by $-\boldsymbol{Q}_{a}^{+}$). Multiplying both sides of (17) from the right with the matrix

$$
\varphi_{q, X^{(-)}}^{-}\left(-\boldsymbol{Q}_{a}^{+}\right)=\int \mathrm{e}^{\boldsymbol{Q}_{a} x} \mathrm{P}\left(-I_{e(q)}^{(-)} \in \mathrm{d} x\right) \quad\left(\text { with } q=a+\lambda^{(+)}\right)
$$

yields

$$
\Phi\left(a+\lambda^{(+)}\right)^{-1}\left[\Phi\left(a+\lambda^{(+)}\right) \boldsymbol{I}+\boldsymbol{Q}_{a}^{+}\right]_{1}=\frac{\lambda^{(+)}}{a+\lambda^{(+)}}(0, \boldsymbol{\alpha}) \varphi_{a+\lambda^{(+)}, X^{(-)}}^{-}\left(-\boldsymbol{Q}_{a}^{+}\right) .
$$

Thus, we find that the first row of $\boldsymbol{Q}_{a}^{+}$is

$$
\left[\boldsymbol{Q}_{a}^{+}\right]_{1}=-\Phi\left(a+\lambda^{(+)}\right) \mathbf{1}_{0}^{\top}+\Phi\left(a+\lambda^{(+)}\right) \frac{\lambda^{(+)}}{a+\lambda^{(+)}}(0, \boldsymbol{\alpha}) \varphi_{a+\lambda^{(+)}, X^{(-)}}^{-}\left(-\boldsymbol{Q}_{a}^{+}\right),
$$

and (16) follows.

We now turn to the proof of Theorem 2.

Proof of Theorem 2(ii). We first determine the form of the process $H$. Since $X^{(-)}$has no positive jumps, $\Delta M_{t}>0$ implies that $\Delta X_{t}^{(+)}>0$ and, similarly, $\mathrm{d} M_{t}^{\mathrm{c}}>0$ implies that $\mathrm{d} M_{t}^{(-)}>0$, where (recall) $M_{t}^{(-)}$denotes the running supremum of $X^{(-)}$up to time $t$. Write $\sigma_{1}, \sigma_{2}, \ldots$ for the jump times of $X^{(+)}$and, for $i=1,2, \ldots$, recursively define the stopping times $G_{i}=\inf \left\{s \geq D_{i-1}: M_{s}=X_{s}\right\}$ and $D_{i}=\inf \left\{\sigma_{j}: \sigma_{j}>G_{i}\right\}$, where $G_{0}=0$ and $D_{0}=\sigma_{1}$. Note that, for $t \in\left[G_{i}, D_{i}\right), M_{t}$ is continuous and $M$ may jump at $G_{i}$. As $X^{(+)}$ is a compound Poisson process with rate $\lambda^{(+)}$, the differences $D_{i}-G_{i}$ are exponentially distributed with parameter $\lambda^{(+)}$and, as $X^{(-)}$has independent, identically distributed increments and $M^{(-)}\left(e\left(\lambda^{(-)}\right)\right)$has an exponential distribution with mean $\Phi\left(\lambda^{(+)}\right)^{-1}, M_{D_{i}}-M_{G_{i}}$ are independent exponential random variables with parameter $\Phi\left(\lambda^{(+)}\right)$. Since we have taken the 
local time, $L$, to be equal to $M^{\mathrm{c}}$, the interarrival time of two jumps of $H$ is $\operatorname{Exp}\left(\Phi\left(\lambda^{(+)}\right)\right)$distributed and, thus, the process $H$ is given by

$$
H(t)=M^{\mathrm{c}}\left(L^{-1}(t)\right)+\sum_{s \leq t} \Delta M\left(L^{-1}(s)\right) 1_{\left\{\Delta M\left(L^{-1}(s)\right)>0\right\}}=t+\sum_{n=1}^{N_{t}} \tilde{U}_{n},
$$

where the $\tilde{U}_{n}$ are independent, identically distributed nonnegative random variables (since $X$ is a Lévy process) and $N_{t}$ is an independent Poisson process with rate $\Phi\left(\lambda^{(+)}\right)$. The jump sizes $\tilde{U}_{n}$ have the same distribution as the overshoot, $X_{T^{+}(0)}$, of $X$ over the level 0 if $X_{0}$ is distributed according to $-A+B$, where $A$ has the distribution

$$
\xi_{0}(\mathrm{~d} x)=\mathrm{P}\left(\left(M^{(-)}-X^{(-)}\right)_{e(\lambda(+))} \in \mathrm{d} x\right)
$$

of the distance of $X^{(-)}$to its supremum at an exponential time $e\left(\lambda^{(+)}\right)$and $B$, independent of $A$, is distributed according to the jump sizes of $X^{(+)}$. Since the upward jumps of $X$ are of phase type, it follows that the distribution of the overshoot $X_{T(0)}$ has an atom at 0 and on $(0, \infty)$ is of (defective) phase type (see, e.g. [5, Proposition 2, p. 90] for a proof). A generator matrix of this phase-type distribution is seen to be given by $\boldsymbol{T}^{(+)}$, with 'initial distribution over the phases' $\hat{\eta}_{0}$ given by the distribution of the underlying Markov process at the moment of crossing. In the equivalent setting of the embedding $(A, Y)=\left(A_{X}, Y_{X}\right)$ of $X, \hat{\eta}_{0}$ thus satisfies

$$
\begin{aligned}
\hat{\boldsymbol{\eta}}_{0}(j) & =\mathrm{P}\left(\tilde{Y}_{0}^{+}=j \mid A_{0} \stackrel{\mathrm{D}}{=} \xi, Y_{0} \stackrel{\mathrm{D}}{=}\left(0, \boldsymbol{\alpha}^{(+)}\right)\right) \\
& =\int_{0}^{\infty}\left(0, \boldsymbol{\alpha}^{(+)}\right) \mathrm{e}^{\boldsymbol{Q}_{0}^{+} x} \xi_{0}(\mathrm{~d} x),
\end{aligned}
$$

where $j=0, \ldots, m^{(+)}$and $\stackrel{\text { D }}{=}$ ' denotes equality in distribution. Thus, the vector $\hat{\eta}_{0}$ satisfies $\boldsymbol{\eta}=\psi_{0}(\boldsymbol{\eta})$ with $\psi_{0}$ as given in (4). In view of Lemma 1 it then follows that $\hat{\boldsymbol{\eta}}_{0}=\boldsymbol{\eta}_{0}$, and (7) has been proved. The rest of the statements of part (ii) directly follow from the independence of $X^{(+)}$and $X^{(-)}$and the general form of the cumulant for bivariate subordinators.

Proof of Theorem 2(i). Note that, on the one hand, since in (18) we showed that $H$ is a compound Poisson process with unit drift, we have

$$
\lim _{s \rightarrow \infty} s^{-1} \kappa^{+}(a, s)=1 .
$$

On the other hand, the form of the Wiener-Hopf factor $\varphi_{a}^{+}$and the fact that $\left|\mathcal{I}^{(+)}\right|=m^{(+)}+1$ (see, e.g. [5, Lemma 1, p. 86]) imply that

$$
\lim _{s \rightarrow-\infty} s \varphi_{a}^{+}(-s)=\prod_{i \in \mathcal{I}^{(+)}} \frac{\rho_{i}(a)}{\operatorname{det}\left(-\boldsymbol{T}^{(+)}\right)} .
$$

Combining this with the fact that $\varphi_{a}(-s)=\kappa^{+}(a, 0) / \kappa^{+}(a, s)$ (see, e.g. [8, Chapter VII.2, p. 166]) we can first identify $\kappa^{+}(a, 0)$ and then $\kappa^{+}(a, s)$.

\section{The fixed-point equation $\eta=\psi_{a}(\eta)$}

In this section, we derive properties of the equation $\eta=\psi_{a}(\eta)$, proving the statements of Lemma 1 and Theorem 1. 


\subsection{Proof of Lemma 1}

Recall from Proposition 3 that there exists a solution to $\boldsymbol{\eta}=\psi_{a}(\boldsymbol{\eta})$ in $\&$.

Since it can be directly verified from the form of $\boldsymbol{M}^{(+)}$that the matrix $\boldsymbol{M}^{(+)}+\boldsymbol{m}^{(+)} \boldsymbol{v}, \boldsymbol{v} \in \&$, is a generator matrix, it follows, by the probabilistic interpretation of $\psi_{a}(\boldsymbol{v})$ derived in the proof of Theorem 2(ii) (see (19)), that $\psi_{a}(\boldsymbol{v})$ is a subprobability vector. Thus, $\psi$ maps $\&$ to $\&$. Furthermore, if in addition $\boldsymbol{v}$ satisfies $\boldsymbol{v}=\psi_{a}(\boldsymbol{v})$, it solves (10) and the uniqueness of the solution to the fixed-point equation follows from Proposition 2 if $\kappa^{\prime}\left(0^{+}\right):=\lim _{s \downarrow 0} s^{-1}[\kappa(s)-\kappa(0)] \geq 0$ or $a>0$. Indeed, in the latter case the generator matrix $\boldsymbol{Q}_{a}$ is transient, whereas in the former case the embedding $A$ of $X$ satisfies $\sup _{t} A_{t}=\infty$.

If $\kappa^{\prime}\left(0^{+}\right)<0$, Proposition 2 does not apply and we need to provide a different argument to establish uniqueness. Following [2], we shall show that this case can be reduced to the case of positive drift using exponential tilting. If $\kappa^{\prime}\left(0^{+}\right)<0$, there exists a positive root, $\gamma$, of the equation $\kappa(s)=0$. Define the tilted measure $\mathrm{P}^{c}$, for any positive constant $c \geq \gamma$ with $\mathrm{E}\left[\mathrm{e}^{c X_{1}}\right]<\infty$, by the Radon-Nikodým derivative

$$
\left.\frac{\mathrm{dP}^{c}}{\mathrm{dP}}\right|_{\mathcal{F}_{t}}=\exp \left(c X_{t}-\kappa(c) t\right), \quad t \geq 0,
$$

and denote by $\kappa^{c}, \varphi_{a}^{-, c}, \Phi^{c}$, and $\eta^{c}$ the respective quantities $\kappa, \varphi_{a}^{-}, \Phi$, and $\eta$ under the measure $\mathrm{P}^{c}$. It is straightforward to check that, for any $\gamma \leq c$ with $\mathrm{E}\left[\mathrm{e}^{c X_{1}}\right]<\infty$,

$$
\kappa^{c}(s)=\kappa(s+c)-\kappa(c), \quad \varphi^{-, c}(s)=\varphi^{-}(s+c), \quad \Phi^{c}(q)=\Phi\left(q+\kappa_{X^{(-)}}(c)\right)-c
$$

for $q \geq 0$ and $\left(\kappa^{c}\right)^{\prime}\left(0^{+}\right)=\kappa^{\prime}(c)>0$. Hence, under $\mathrm{P}^{c}$ the process $X$ has a positive drift and uniqueness will follow once we have shown that the equation $\boldsymbol{\eta}=\psi_{a}(\boldsymbol{\eta})$ can be formulated in terms of quantities of the process $X$ under the tilted measure $\mathrm{P}^{c}$. The first step in this direction is the next result (from [1]), which shows that, under the tilted measure $\mathrm{P}^{c}$, the jumps of $X^{(+)}$ remain of phase type.

Lemma 2. Under $\mathrm{P}^{c}$, the jumps of $X^{(+)}$are of phase type with representation given by

$$
\left(\lambda^{(+, c)}, \boldsymbol{\alpha}^{(+, c)}, \boldsymbol{T}^{(+, c)}\right)=\left(\lambda^{(+)} \hat{F}^{(+)}[-c], \boldsymbol{\alpha}^{(+)} \boldsymbol{\Delta} / \hat{F}^{(+)}[-c], \boldsymbol{\Delta}^{-1} \boldsymbol{T}^{(+)} \boldsymbol{\Delta}+c \boldsymbol{I}\right),
$$

where $\boldsymbol{\Delta}$ is the diagonal matrix with the components, $k_{j}$, of $\boldsymbol{k}=\left(c \boldsymbol{I}-\boldsymbol{T}^{(+)}\right)^{-1} \boldsymbol{t}^{(+)}$on the diagonal. Furthermore, $\boldsymbol{t}^{(+, c)}=\boldsymbol{\Delta}^{-1} \boldsymbol{t}^{(+)}$.

By choosing the killing rate

$$
a^{c}=a+\lambda^{(+)}\left(1-\hat{F}^{(+)}[-c]\right),
$$

noting that $a+\lambda^{(+)}=a^{c}+\lambda^{(+, c)}$, and recalling the form of $\boldsymbol{M}^{(+)}$from (5), we deduce that, under $\mathrm{P}^{c}$, the generator matrix of the upcrossing ladder process $\tilde{Y}^{+}$is given by

$$
\boldsymbol{Q}^{+, c}=\boldsymbol{M}^{(+), c}+\boldsymbol{m}^{(+), c} \boldsymbol{\eta}_{a}^{c}
$$

where

$$
\begin{aligned}
\boldsymbol{M}^{(+), c} & =\left(\begin{array}{cc}
-\Phi\left(a+\lambda^{(+)}+\kappa_{X^{(-)}}(c)\right)+c & \mathbf{0} \\
\boldsymbol{\Delta}^{-1} \boldsymbol{t}^{(+)} & \boldsymbol{\Delta}^{-1} \boldsymbol{T}^{(+)} \boldsymbol{\Delta}+c \boldsymbol{I}
\end{array}\right), \\
\boldsymbol{m}^{(+), c} & =\left(\Phi\left(a+\lambda^{(+)}+\kappa_{X^{(-)}}(c)\right)-c, \mathbf{0}\right)^{\top},
\end{aligned}
$$


and $\boldsymbol{\eta}_{a}^{c}$ satisfies $\boldsymbol{\eta}=\psi_{a}^{c}(\boldsymbol{\eta})$ with $\psi_{a}^{c}$ as defined in (4) but with $a$ replaced by $a^{c}$ and all other quantities replaced by ones 'tilted' under the measure $\mathrm{P}^{c}$. Next, we provide the link between $\psi_{a}$ and $\psi_{a}^{c}$. Writing $\boldsymbol{\Delta}_{1}=\operatorname{diag}\left(1, k_{1}, \ldots, k_{m}(+)\right)$ and using Lemma 2 , it follows that

$$
\begin{aligned}
\psi_{a}(\boldsymbol{\eta}) & =\frac{\lambda^{(+)}}{a+\lambda^{(+)}}\left(0, \boldsymbol{\alpha}^{(+)}\right) \varphi_{X^{(-)}}^{-}\left(-\boldsymbol{M}^{(+)}-\boldsymbol{m}^{(+)} \boldsymbol{\eta}\right) \\
& =\frac{\lambda^{(+)} \hat{F}^{(+)}[-c]}{a+\lambda^{(+)}}\left(0, \boldsymbol{\alpha}^{(+), c}\right) \boldsymbol{\Delta}_{1}^{-1} \varphi_{X^{(-)}}^{-, c}\left(-\boldsymbol{M}^{(+)}-\boldsymbol{m}^{(+)} \boldsymbol{\eta}-c \boldsymbol{I}\right) \\
& =\frac{\lambda^{(+), c}}{a+\lambda^{(+)}}\left(0, \boldsymbol{\alpha}^{(+), c}\right) \varphi_{X^{(-)}}^{-, c}\left(-\boldsymbol{\Delta}_{1}^{-1}\left(\boldsymbol{M}^{(+)}+c \boldsymbol{I}\right) \boldsymbol{\Delta}_{1}-\boldsymbol{\Delta}_{1}^{-1} \boldsymbol{m}^{(+)} \eta \boldsymbol{\Delta}_{1}\right) \boldsymbol{\Delta}_{1}^{-1} \\
& =\frac{\lambda^{(+), c}}{a^{c}+\lambda^{(+), c}}\left(0, \boldsymbol{\alpha}^{(+), c}\right) \varphi_{X^{(-)}}^{-, c}\left(-\boldsymbol{M}^{(+), c}-\boldsymbol{m}^{(+), c} \tilde{\boldsymbol{\eta}}\right) \boldsymbol{\Delta}_{1}^{-1}
\end{aligned}
$$

where $\tilde{\boldsymbol{\eta}}=\boldsymbol{\eta} \boldsymbol{\Delta}_{1}$. Since $\left(\kappa^{c}\right)^{\prime}\left(0^{+}\right)>0$ for $c \geq \gamma$, Proposition 2 implies that, for these values of c. $\tilde{\boldsymbol{\eta}}=\psi_{a}^{c}(\tilde{\boldsymbol{\eta}})$ has a unique solution, $\tilde{\boldsymbol{\eta}}$, in $\&$. Since $\psi_{a}^{c}(\tilde{\boldsymbol{\eta}})$ is equal to $\psi_{a}(\boldsymbol{\eta}) \boldsymbol{\Delta}_{1}$ and the elements of $\boldsymbol{\Delta}_{1}$ are positive and not greater than one, any solution $\boldsymbol{\eta} \in \delta$ to $\psi_{a}(\boldsymbol{\eta})=\boldsymbol{\eta}$ gives rise to a solution $\tilde{\boldsymbol{\eta}}=\boldsymbol{\eta} \boldsymbol{\Delta}_{1} \in \&$ to $\tilde{\boldsymbol{\eta}}=\psi_{a}^{c}(\tilde{\boldsymbol{\eta}})$. Thus, if $\kappa^{\prime}\left(0^{+}\right)<0$, it follows that $\boldsymbol{\eta}_{a}$ is the unique subprobability vector that solves $\boldsymbol{\eta}=\psi_{a}(\boldsymbol{\eta})$.

\subsection{Proof of Theorem 1(i)}

Write $\boldsymbol{\eta} \leq \boldsymbol{\eta}^{\prime}$ if $\boldsymbol{\eta}^{\prime}-\boldsymbol{\eta}$ is nonnegative (coordinatewise). We claim that $\psi_{a}$ satisfies the following monotonicity property: if $\boldsymbol{\eta} \leq \boldsymbol{\eta}^{\prime}$ then $\psi_{a}(\boldsymbol{\eta}) \leq \psi_{a}\left(\boldsymbol{\eta}^{\prime}\right)$. Let $\boldsymbol{P}$ and $\boldsymbol{P}^{\prime}$ be the transition matrices of the Markov chains with respective generators given by $\boldsymbol{G}(\boldsymbol{\eta})=\boldsymbol{M}^{(+)}+\boldsymbol{m}^{(+)} \boldsymbol{\eta}$ and $\boldsymbol{G}\left(\boldsymbol{\eta}^{\prime}\right)$. Then the matrix $\boldsymbol{D}=\boldsymbol{P}^{\prime}-\boldsymbol{P}$ satisfies the matrix differential equation

$$
\dot{\boldsymbol{D}}=\boldsymbol{D} \boldsymbol{G}(\boldsymbol{\eta})+\boldsymbol{P}^{\prime} \boldsymbol{m}^{(+)}\left(\boldsymbol{\eta}^{\prime}-\boldsymbol{\eta}\right), \quad \boldsymbol{D}(0)=\boldsymbol{O},
$$

where $\dot{\boldsymbol{D}}=\mathrm{d} \boldsymbol{D}(t) / \mathrm{d} t$ and $\boldsymbol{O}$ denotes the zero matrix. The solution to this equation is

$$
\boldsymbol{D}(t)=\int_{0}^{t} \boldsymbol{P}^{\prime}(s) \boldsymbol{m}^{(+)}\left(\boldsymbol{\eta}^{\prime}-\boldsymbol{\eta}\right) \mathrm{e}^{(t-s) \boldsymbol{G}(\boldsymbol{\eta})} \mathrm{d} s .
$$

Hence, coordinatewise, $\boldsymbol{D}$ is nonnegative and, since it holds that

$$
\psi_{a}(\boldsymbol{\eta})=\frac{\lambda^{(+)}}{a+\lambda^{(+)}}\left(0, \boldsymbol{\alpha}^{(+)}\right) \int_{0}^{\infty} \mathrm{e}^{x \boldsymbol{G}(\boldsymbol{\eta})} \mathrm{P}\left(-I_{e(a)}^{(-)} \in \mathrm{d} x\right),
$$

the claim follows. In particular, it follows that $f_{a}(\boldsymbol{\eta}) \leq f_{a}\left(\boldsymbol{\eta}^{\prime}\right)$.

Starting with $\boldsymbol{\eta}^{(0)}=\mathbf{0}$ and setting $\boldsymbol{\eta}^{(n+1)}=\psi_{a}\left(\boldsymbol{\eta}^{(n)}\right)$ generates a sequence $\left(\boldsymbol{\eta}^{(n)}\right)_{n}$ in $\&$ which is coordinatewise nondecreasing. Thus, the sequence has a limit in $\delta$, say $\boldsymbol{v}$, and by the continuity of $\psi_{a}$ it follows that $\boldsymbol{v}=\psi_{a}(\boldsymbol{v})$. The uniqueness due to Lemma 1 implies that $\boldsymbol{v}=\boldsymbol{\eta}_{a}$.

\subsection{Proof of Theorem 1(ii)}

The proof of Theorem 1(ii) follows from the next result.

Proposition 4. Let $a>0$. If $\kappa_{X^{(-)}}^{\prime}\left(0^{+}\right) \geq 0$ then the map $\psi_{a}$ is a contraction. If $\kappa_{X^{(-)}}^{\prime}\left(0^{+}\right)<0$ and $\hat{F}^{(+)}[-\Phi(0)]<1+a / \lambda^{(+)}$then the map $\psi_{a}^{\Phi(0)}$ is a contraction. 
Proof. Write $\mathbf{1}_{i}$ for the column vector with a 1 in state $i$ and 0 s elsewhere, and let

$$
\eta_{t}=\eta+t\left(\eta^{\prime}-\eta\right)
$$

with $t \in[0,1]$, be the convex combination of $\boldsymbol{\eta}, \boldsymbol{\eta}^{\prime} \in$ \&. Since

$$
\varphi_{i}: t \mapsto(0, \boldsymbol{\alpha}) \mathrm{e}^{x\left(\boldsymbol{M}^{(+)}+\boldsymbol{m}^{(+)} \boldsymbol{\eta}_{t}\right)} \mathbf{1}_{i}
$$

is differentiable, the mean value theorem implies that, for some $s \in[0,1]$,

$$
\begin{aligned}
\left|\varphi_{i}(1)-\varphi_{i}(0)\right| & =\left|(0, \boldsymbol{\alpha}) \mathrm{e}^{x\left(\boldsymbol{M}^{(+)}+\boldsymbol{m}^{(+)} \boldsymbol{\eta}_{s}\right)} \boldsymbol{m}^{(+)}\left(\boldsymbol{\eta}-\boldsymbol{\eta}^{\prime}\right) x \mathbf{1}_{i}\right| \\
& =(0, \boldsymbol{\alpha}) \mathrm{e}^{x\left(\boldsymbol{M}^{(+)}+\boldsymbol{m}^{(+)} \boldsymbol{\eta}_{s}\right)} \mathbf{1}_{0} \Phi(a)\left|\left(\boldsymbol{\eta}-\boldsymbol{\eta}^{\prime}\right)_{i}\right| x \\
& \leq x \Phi(a)\left|\left(\boldsymbol{\eta}-\boldsymbol{\eta}^{\prime}\right)_{i}\right|,
\end{aligned}
$$

where we have used the form of $\boldsymbol{m}^{(+)}$and the fact that in the second line the first factor is the probability that some Markov chain is in state 0 at time $x$. By letting $\lambda=\lambda^{(+)}$and $\boldsymbol{\alpha}=\boldsymbol{\alpha}^{(+)}$, we obtain

$$
\begin{aligned}
\left\|\psi_{a}\left(\boldsymbol{\eta}^{\prime}\right)-\psi_{a}(\boldsymbol{\eta})\right\| & =\sum_{i}\left|\varphi_{i}(1)-\varphi_{i}(0)\right| \\
& \leq \sum_{i} \frac{\lambda}{a+\lambda} \int_{0}^{\infty} x \Phi(a)\left|\left(\boldsymbol{\eta}-\boldsymbol{\eta}^{\prime}\right)_{i}\right| \mathrm{P}\left(-I_{e(a)}^{(-)} \in \mathrm{d} x\right) \\
& =\frac{\lambda}{a+\lambda} \mathrm{E}\left[-I_{e(a)}^{(-)}\right] \Phi(a)\left\|\boldsymbol{\eta}-\boldsymbol{\eta}^{\prime}\right\| .
\end{aligned}
$$

Since

$$
\kappa_{X^{(-)}}^{\prime}\left(0^{+}\right) / a=\mathrm{E}\left[X_{e(a)}^{(-)}\right]=\mathrm{E}\left[X_{e(a)}^{(-)}-I_{e(a)}^{(-)}\right]+\mathrm{E}\left[I_{e(a)}^{(-)}\right]=\frac{1}{\Phi(a)}+\mathrm{E}\left[I_{e(a)}^{(-)}\right]
$$

it follows that $\mathrm{E}\left[-I_{e(a)}^{(-)}\right] \Phi(a) \leq 1$ if and only if $\mathrm{E}\left[X_{1}^{(-)}\right] \geq 0$.

Let us now turn to the case in which $\kappa_{X^{(-)}}^{\prime}\left(0^{+}\right)<0$ when $\Phi(0)>0$. Since $\mathrm{E}\left[\mathrm{e}^{\Phi(0) X_{1}}\right]$ is assumed to be finite and $\Phi(0) \geq \gamma\left(\right.$ since $\left.\kappa(\Phi(0))=\lambda^{(+)}\left(\hat{F}^{(+)}[-\Phi(0)]-1\right)>0\right)$, it follows both that the exponential tilting with $\underset{\Phi(0)}{c}=\Phi(0)$ is well defined and that $\kappa_{X^{(-)}}^{\Phi(0) \prime}\left(0^{+}\right)>0$. By following the above reasoning with $\psi_{a}^{\Phi(0)}$ instead of $\psi_{a}$, we deduce that

$$
\begin{aligned}
\left|\left(\psi_{a}\left(\boldsymbol{\eta}^{\prime}\right)-\psi_{a}(\boldsymbol{\eta})\right)_{i}\right| & =\left|\left(\psi_{a}^{\Phi(0)}\left(\tilde{\boldsymbol{\eta}}^{\prime}\right)-\psi_{a}^{\Phi(0)}(\tilde{\boldsymbol{\eta}})\right)_{i}\right|\left(\boldsymbol{\Delta}_{1}\right)_{i}^{-1} \\
& \leq \frac{\lambda \hat{F}^{(+)}[-\Phi(0)]}{\lambda+a} \mathrm{E}^{\Phi(0)}\left[-I_{e(a)}^{(-)}\right](\Phi(a)-\Phi(0))\left|\left(\tilde{\boldsymbol{\eta}}^{\prime}-\tilde{\boldsymbol{\eta}}\right)_{i}\right|\left(\boldsymbol{\Delta}_{1}\right)_{i}^{-1} \\
& \leq \frac{\lambda \hat{F}^{(+)}[-\Phi(0)]}{\lambda+a} \mathrm{E}^{\Phi(0)}\left[-I_{e(a)}^{(-)}\right](\Phi(a)-\Phi(0))\left|\left(\boldsymbol{\eta}^{\prime}-\boldsymbol{\eta}\right)_{i}\right|,
\end{aligned}
$$

where $\mathrm{E}^{\Phi(0)}\left[-I_{e(a)}^{(-)}\right](\Phi(a)-\Phi(0))<1$ since $\kappa_{X^{(-)}}^{\Phi(0)}\left(0^{+}\right)>0$ and $\lambda \hat{F}^{(+)}[-\Phi(0)] /(\lambda+a)<1$ since $\hat{F}^{(+)}[-\Phi(0)]<(\lambda+a) / \lambda$.

This completes the proof of Proposition 4.

Note that (20), together with the definition of $f_{a}(\boldsymbol{\eta})$, implies that

$$
\left|f_{a}\left(\boldsymbol{\eta}_{a}\right)-f_{a}\left(\boldsymbol{\eta}^{(n)}\right)\right| \leq a^{-1} k \Phi(a)\left\|\boldsymbol{\eta}_{a}-\boldsymbol{\eta}^{(n)}\right\|=a^{-1} k \Phi(a)\left\|\psi_{a}\left(\boldsymbol{\eta}_{a}\right)-\psi_{a}\left(\boldsymbol{\eta}^{(n-1)}\right)\right\| .
$$

Combining this with (21), (22), and (23) completes the proof of Theorem 1(ii). 


\section{Acknowledgements}

The author would like to thank Florin Avram for inspiring and stimulating conversations and Ernst Eberlein for useful comments. This research was supported by the Nuffield Foundation, grant no. NAL/00761/G.

\section{References}

[1] Asmussen, S. (1989). Exponential families generated by phase-type distributions and other Markov lifetimes. Scand. J. Statist. 16, 319-334.

[2] Asmussen, S. (1992). Phase-type representations in random walk and queueing problems. Ann. Prob. 20, 772789.

[3] Asmussen, S. (2000). Ruin Probabilities. World Scientific, River Edge, NJ.

[4] Asmussen, S. (2003). Applied Probability and Queues, 2nd edn. Springer, New York.

[5] Asmussen, S., Avram, F. and Pistorius, M. R. (2004). Russian and American put options under phase-type Lévy models. Stoch. Process. Appl. 109, 79-111.

[6] Asmussen, S., Madan, D. P. and Pistorius, M. R. (2005). Pricing equity default swaps under the CGMY Lévy model. Submitted.

[7] Barndorff-Nielssen, O. (2000). Processes of normal inverse Gaussian type. Finance Stoch. 2, 41-68.

[8] Bertoin, J. (1996). Lévy Processes. Cambridge University Press.

[9] Bingham, N. H. (1975). Fluctuation theory in continuous time. Adv. Appl. Prob. 7, 705-766.

[10] Carr, P., Geman, H., Madan, D. P. and Yor, M. (2002). The fine structure of asset returns: an empirical investigation. J. Business 75, 305-332.

[11] Eberlein, E. and Keller, U. (1995). Hyperbolic distributions in finance. Bernoulli 1, 281-299.

[12] Jacod, J. And Shiryaev, A. N. (2003). Limit Theorems for Stochastic Processes, 2nd edn. Springer, Berlin.

[13] Kou, S. G. (2002). A jump diffusion model for option pricing. Manag. Sci. 48, 1086-1101.

[14] London, R. R., McKean, H. P., Rogers, L. C. G. And Williams, D. (1982). A martingale approach to some Wiener-Hopf problems. I, II. In Seminar on Probability, XVI (Lecture Notes Math. 920), Springer, Berlin, pp. 41-90.

[15] MordecKI, E. (2002). Optimal stopping and perpetual options for Lévy processes. Finance Stoch. 6, $473-493$.

[16] Mordecki, E. (2002). The distribution of the maximum of a Lévy process with positive jumps of phase-type. Theory Stoch. Process. 8, 309-316.

[17] Neuts, M. F. (1981). Matrix-Geometric Solutions in Stochastic Models. Johns Hopkins University Press, Baltimore, MD.

[18] Neuts, M. F. (1989). Structured Stochastic Matrices of M/G/1 Type and Their Applications. Marcel Dekker, New York. 